\title{
CONCENTRACIONES ATMOSFERICAS DE PROPAGULOS FUNGICOS EN VIÑEDOS DEL RIBEIRO (GALICIA, ESPAÑA)
}

\author{
(Atmospheric concentrations of fungal propagules in vineyards of Ribeiro valley \\ (Galicia, Spain))
}

\author{
*Y. Albelda, *F. J. Rodríguez-Rajo, *V. Jato \& **M.J. Aira \\ * Departamento de Biología Vegetal y Ciencias del Suelo, \\ Facultad de Ciencias de Ourense, Universidad de Vigo (España) \\ ** Departamento de Botánica, Facultad de Farmacia, Universidad de Santiago (España)
}

Palabras clave: Aerosporas, viñedo, modelos predictivos, Botrytis, Uncinula, Plasmopara, España

Key words: Aerospores, vineyard, predictive models, Botrytis, Uncinula, Plasmopara, Spain

\section{RESUMEN}

Con el fin de conocer la incidencia de la podredumbre gris, el oidio y el mildiu y contribuir a paliar sus efectos en la vid mediante la aplicación de un tratamiento fitosanitario adecuado, se ha realizado un estudio de la concentración atmosférica de los conidios de Botrytis y Uncinula y los esporangios de Plasmopara, en un viñedo de la provincia de Ourense (Galicia, España), durante la época vegetativa del cultivo en el año 2004. De los tres fitopatógenos estudiados, se han encontrado cantidades importantes de Botrytis, con un total de 6.519 conidios y Uncinula con 1.554 conidios, ambos presentes en la atmósfera preferentemente entre las 19-20 h. La concentración de esporangios de Plasmopara, ha sido inferior, con un total de 311 esporangios, registrándose los picos más elevados entre las 17-18 h. A partir de los datos de las concentraciones diarias de estos propágulos, se ha analizado estadísticamente la influencia de las variables meteorológicas sobre su presencia en la atmósfera, destacando positivamente la humedad absoluta y la temperatura de rocío, sobre la concentración de conidios de Botrytis y esporangios de Plasmopara y la temperatura máxima para los conidios de Uncinula. Se han desarrollado modelos de predicción de los niveles de estos propágulos, que podrían ser de gran utilidad para la valoración del riesgo de infección.

\section{INTRODUCCION}

El gran interés económico alcanzado en las últimas décadas por el cultivo de la vid en Galicia, y en concreto por los vinos con Denominación de Origen del Ribeiro, ha propiciado la realización de diversos trabajos que tienen

\section{ABSTRACT}

With the purpose of knowing the incidence of grey mould, powdery mildew and downy mildew and thus minimize their effect on vineyards through the application of a suitable fungicide treatment, we studied the atmospheric concentrations of conidia of Botrytis and Uncinula together with the sporangia of Plasmopara in a vineyard located in the province of Ourense (Galicia, Spain), in the year 2004, during the vegetative stage of the growing. Among the three fitopathogenous examined, a high number of Botrytis, with a total of 6.519 conidia and Uncinula, with 1,554 conidia was detected, both being present in the atmosphere mainly within $19-20 \mathrm{~h}$. The concentration of Plasmopara sporangia has been lower, with a total of 311, the highest peak being recorded within 17-18h. Based on data collected from daily concentrations of these propagules, the influence of meteorological fluctuations on their presence in the atmosphere has been statistically analysed, and it has been concluded that absolute humidity as well as temperature of dew have positive effects on the concentration of Botrytis conidia and Plasmopara sporangia whereas maximum temperature shows similar results in the case of Uncinula conidia. Predictive models regarding the levels of these propagules were developed in order to ascertain risk of infection.

como objetivo la mejora de la calidad de las condiciones del cultivo y en consecuencia, la de los caldos cosechados (Mansilla, 1991; Fernández, 2005). Uno de los aspectos de mayor interés para lograr dichos objetivos, consiste en el estudio de las enfermedades fúngicas que son comunes 
en nuestra región como la podredumbre gris, el oidio o el mildiu, a través del control de los propágulos fúngicos en la atmósfera de los viñedos (Díaz et al., 1997; Díaz, 1999; Rodriguez-Rajo et al., 2002). Este tipo de estudios permite valorar el riesgo de extensión de la enfermedad y utilizar tratamientos fitosanitarios para combatirla, de forma equilibrada y eficaz.

En el presente estudio se ha analizado la incidencia de las tres enfermedades citadas en un viñedo de la comarca del Ribeiro (Galicia, España), durante la época vegetativa del cultivo, en el año 2004. Dicho estudio se ha llevado a cabo, recogiendo por métodos aerobio-lógicos, los conidios de Botrytis cinerea Pers. ex Nocca \& Balb. y de Uncinula necator (Schw.) Burr. y los esporangios de Plasmopara viticola (Berk. \& Curt.) Berl. \& de Toni.

Asimismo se ha realizado un seguimiento fenológico de la planta y un registro de las condiciones microclimáticas del cultivo, con el fin de evaluar la influencia de los principales parámetros meteorológicos sobre la concentración de los propágulos fúngicos, para finalmente proponer un modelo que permita predecir sus niveles para años futuros.

\section{MATERIALES Y METODOS}

El estudio se llevó a cabo en la localidad de Cenlle, en la comarca del Ribeiro de la provincia de Ourense (Figura 1). El cultivo ocupa 2.800 hectáreas, con diversas variedades de Vitis vinifera (treixadura, loureira y godello), que producen unos $17.000 \mathrm{Kg}$ de uva en cada cosecha. La recogida de los propágulos fúngicos se realizó mediante un captador volumétrico modelo Lanzoni VPPS2000, situado en la zona central del cultivo a una altura de 2 metros sobre el nivel del suelo, para evitar que la captura se viese obstaculizada por el crecimiento de las viñas.

El período de estudio abarcó desde el momento en el que comenzaron a desarrollarse las hojas de la planta (15 de abril) hasta la vendimia (22 de septiembre) del año 2004, es decir durante un total de 161 días. Se han realizado asimismo observaciones periódicas en el campo, para detectar la aparición de sintomatología de las infecciones y anotar las fechas correspondientes a las fases fenológicas.

Para la realización del estudio del comportamiento intradiario se tuvieron en cuenta aquellos días con concentraciones medias diarias, iguales o superiores a la media alcanzada durante el período de muestreo. Dentro de estos días, se seleccionaron aquellos en los que no se registraron precipitaciones, calculándose con ellos la media para cada dos horas, expresada en porcentajes (Galán et al., 1991).

Los datos meteorológicos fueron obtenidos de la estación Cenlle situada a escasos metros del viñedo.

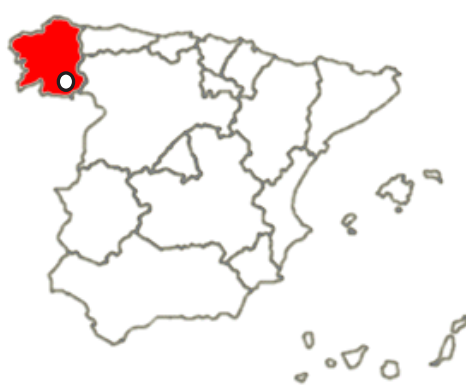

Figura 1.- Localización de Cenlle en Galicia (España)

Con el objeto de comprobar el grado de asociación que existe entre las concentraciones de conidios de Botrytis y Uncinula y esporangios de Plasmopara, con las principales variables climáticas, se ha realizado un test no paramétrico de correlación de Spearman, al comprobarse que los datos no siguen modelos de distribución normal, calculando el grado de significación para los intervalos de confianza del $90 \%(*), 95 \%(* *)$ y del $99 \%(* * *)$. De esta forma se han correlacionado los datos diarios de la concentración de propágulos con los correspondientes a los obtenidos 1, 2 y 3 días anteriores. También se han correlacionado las concentraciones fúngicas (variable dependiente) con los principales parámetros meteorológicos (variable independiente), tanto los registrados en el mismo día como en 1, 2 y 3 días anteriores.

Finalmente, con el fin de establecer un modelo que permita realizar predicciones de la concentración de estos propágulos, se realizó un análisis de regresión múltiple. Como estimadores del modelo se utilizaron aquellos parámetros que en el análisis de correlación de Spearman proporcionaron un coeficiente de correlación positivo y elevado, utilizando asimismo como predictor la concentración de conidios o esporangios del día anterior.

\section{RESULTADOS}

La presencia de conidios de Botrytis y Uncinula en la atmósfera ha sido constante durante todo el período de muestreo, mientras que los esporangios de Plasmopara estuvieron ausentes durante el mes de abril. Botrytis fue el que alcanzó el valor total más elevado con 6.519 conidios, mientras que la presencia de Uncinula y Plasmopara fue menor, alcanzando 1.554 conidios y 311 esporangios respectivamente. La máxima concentración mensual de conidios de Botrytis se registró en septiembre, con 2.901 conidios aunque también presentó niveles considerables en mayo y junio, superando los 1.000 conidios/mes. Por su parte los conidios de Uncinula, tienen una clara representación durante la primavera y principios del verano, con el máximo mensual localizado en junio, con 825 conidios. Finalmente los esporangios de Plasmopara, muestran su máximo mensual en septiembre 
Tabla 1.- Valores totales, mensuales y diarios de los propágulos de Botrytis, Uncinula y Plasmopara

\begin{tabular}{|c|c|c|c|c|c|}
\hline \multirow{7}{*}{ Botrytis } & & $\begin{array}{l}\text { Total } \\
\text { mensual }\end{array}$ & $\begin{array}{c}\text { Máximo } \\
\text { diario }\end{array}$ & $\begin{array}{c}\text { Día de } \\
\text { máximo }\end{array}$ & $\begin{array}{c}\text { Total } \\
\text { período }\end{array}$ \\
\hline & 15 abril & 6 & 2 & 27 & \multirow{6}{*}{6.519} \\
\hline & mayo & 1.158 & 219 & 27 & \\
\hline & junio & 1.034 & 93 & 5 & \\
\hline & julio & 742 & 83 & 17 & \\
\hline & agosto & 678 & 54 & 16 & \\
\hline & 22 septiembre & 2.901 & 368 & 16 & \\
\hline \multirow{6}{*}{ Uncinula } & 15 abril & 10 & 31 & 30 & \multirow{6}{*}{1.554} \\
\hline & mayo & 267 & 31 & 30 & \\
\hline & junio & 852 & 120 & 14 & \\
\hline & julio & 215 & 26 & 6 & \\
\hline & agosto & 80 & 17 & 27 & \\
\hline & 22 septiembre & 130 & 15 & 16 & \\
\hline \multirow{6}{*}{ Plasmopara } & 15 abril & $\mathbf{0}$ & $\mathbf{0}$ & $\mathbf{0}$ & \multirow{6}{*}{311} \\
\hline & mayo & 26 & 4 & $20 / 30$ & \\
\hline & junio & 83 & 13 & 8 & \\
\hline & julio & 53 & 14 & 4 & \\
\hline & agosto & 38 & 11 & 15 & \\
\hline & 22 septiembre & 111 & 18 & 22 & \\
\hline
\end{tabular}

(111 esporangios), siendo también notable su presencia en la atmósfera durante el mes de junio (Tabla 1).

En cuanto a las concentraciones máximas diarias, es nuevamente Botrytis el que alcanzó el valor más elevado, con 368 conidios $/ \mathrm{m}^{3}$ el día 16 de septiembre (Figura 2). Uncinula presentó un pico máximo de 120 conidios $/ \mathrm{m}^{3}$ el día 14 de junio (Figura 3) y finalmente Plasmopara alcanzó un máximo de 18 esporangios $/ \mathrm{m}^{3} \mathrm{el}$ 22 de septiembre (Figura 4).

Aunque con diferentes valores de representación, los modelos de comportamiento intradiurno obtenidos, muestran un incremento de la concentración de propágulos durante la tarde (19-20 horas en el caso de Botrytis y Uncinula y 17-18 horas en Plasmopara) y descenso progresivo en las primeras horas del día, hasta alcanzar los valores más bajos por la mañana (Figura 5) .

La influencia que las variables meteorológicas ejercen sobre la concentración de propágulos no es homogénea para los diferentes tipos fúngicos estudiados (Tabla 2). Los valores del coeficiente de correlación en el caso de los conidios de Botrytis fueron positivas (nivel de significación del 99\%) con las concentraciónes de conidios, humedad, temperatura mínima y punto de rocío, considerando tanto los valores del mismo día como los de los 3 días anteriores. La única variable con correlación negativa fue la precipitación y la velocidad del viento.

En el caso de Uncinula, la concentración de conidios, temperatura máxima y humedad relativa, considerando tanto los valores del mismo día como los 3 días anteriores y la temperatura mínima del mismo día, muestran correlación positiva (99\% de significación). La correlación fue negativa y con el mismo nivel de significación con la precipitación. Para los esporangios de Plasmopara se ha registrado correlación positiva (nivel de significación del 99\%) con la concentración de esporangios, temperatura mínima, humedad absoluta y punto de rocío, en lo que respecta tanto los valores del mismo día como los 3 días anteriores, y con la temperatura máxima del día anterior. La correlación presentó un signo negativo (nivel de significación del 99\%) con la precipitación del día anterior.

Finalmente a partir de las correlaciones obtenidas y con el fin de obtener ecuaciones que permitan predecir la concentración de esporas en la atmósfera, se ha realizado el análisis de regresión utilizando como variables independientes en el caso de Botrytis y Plasmopara, la concentración de propágulos, la humedad absoluta y la temperatura de rocío, en todos los valores del día anterior (Tablas 3 y 5). Este análisis de tipo polinomial, resultó significativo con unos valores F de 41.093 (g.l. $=3.135$ y $\mathrm{p}=0,000$ ) y de 3.135 (g.l. $=3.135$ y $\mathrm{p}=0,000$ ) respectivamente. Las rectas de regresión explican un $46.6 \%$ de la variabilidad de la concentración de conidios de Botrytis y un 13.5\% de la variabilidad de esporangios de Plasmopara. En el caso de Uncinula, las variables independientes selecciona-das fueron la concentración de conidios del día anterior, la humedad relativa de tres días antes y la temperatura máxima del día anterior (Tabla 4). La recta de regresión propuesta explica un $43.6 \%$ de la variabilidad de la concentración de conidios de dicho género. 


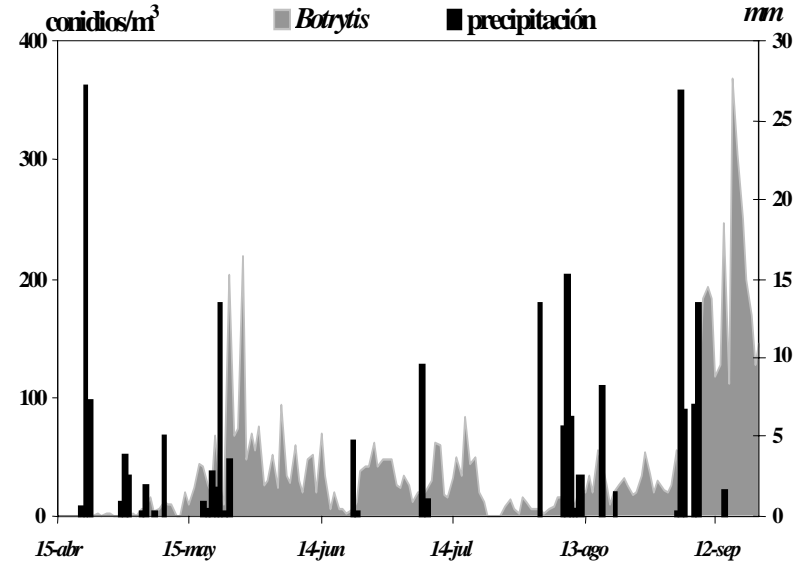

Figura 2.- Concentración de conidios de Botrytis y precipitaciones registradas durante el período.

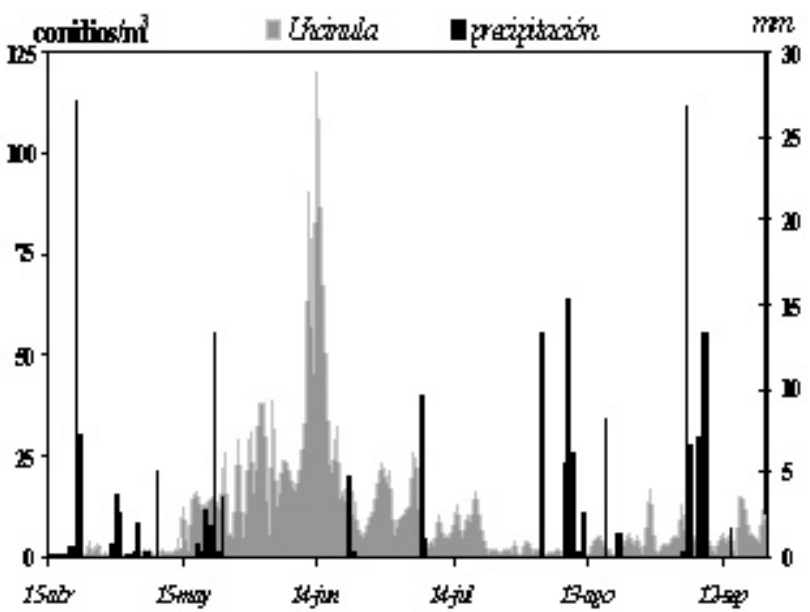

Figura 3.- Concentración de conidios de Uncinula y precipitaciones registradas durante el período.

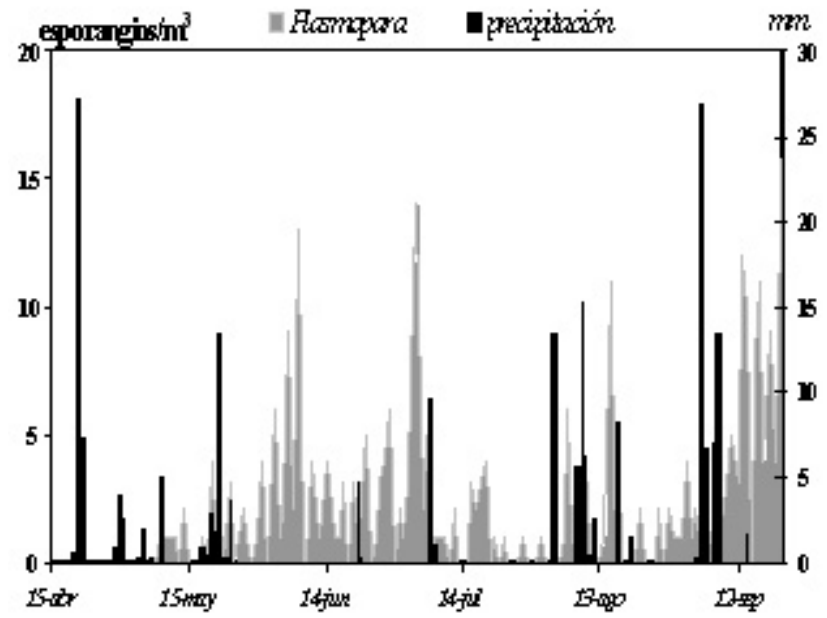

Figura 4.- Concentración de esporangios de Plasmopara y precipitaciones registradas durante el período.
Tabla 2.- Corrrelación entre la concentración de propágulos en el período de estudio y su concentración de los días anteriores así como las principales variables meteorológicas utilizando el factor de correlación de Spearman $(* \mathbf{p}<0.1 ; * * \mathbf{p}<0.05 ; * * * \mathbf{p}<0.001)$. Se han considerado también los valores de los parámetros meteorológicos los tres días anteriores al registro.

\begin{tabular}{|c|c|c|c|}
\hline & Botrytis & Uncinula & $\begin{array}{c}\text { Plasmo- } \\
\text { para }\end{array}$ \\
\hline Propágulos -1 & $0.808^{\star \star \star}$ & $0.707^{\star \star \star}$ & $0.353^{\text {** }}$ \\
\hline Propágulos -2 & $0.719^{\star \star \star}$ & $0.729^{\star \star \star}$ & $0.341^{\text {k* }}$ \\
\hline Propágulos -3 & $0.719^{\star \star \star}$ & $0.652^{\star \star \star}$ & $0.297^{\text {*A }}$ \\
\hline Precipitación & $-0.233^{\star \star \star}$ & $-0.301^{\star \star \star ~}$ & $-0.151^{\star}$ \\
\hline Precipitación -1 & $-0.198^{\star \star}$ & $-0.261^{\star \star \star A}$ & $-0.246^{\star \star \star x}$ \\
\hline Precipitación-2 & ns & $-0.278^{\star \star \star}$ & ns \\
\hline Precipitación -3 & ns & $-0.238^{\star \star \star}$ & $-0.187^{\star \star}$ \\
\hline Velocidad Viento & $-0.149^{\star}$ & ns & ns \\
\hline Viento - 1 & $-0.174^{\text {^夫 }}$ & ns & $-0.140^{\star}$ \\
\hline Viento -2 & $-0.136^{\star}$ & ns & ns \\
\hline Viento -3 & ns & ns & ns \\
\hline Humed absoluta & $0.492^{\star \star \star}$ & ns & $0.378^{\star \star \star}$ \\
\hline H. absoluta - 1 & $0.463^{\star \star \star}$ & ns & $0.336^{\star \star \star}$ \\
\hline H. absoluta-2 & 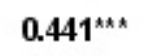 & ns & $0.333^{\star \star \star}$ \\
\hline H. absoluta -3 & $0.405^{\star \star \star}$ & ns & $0.322^{\star \star \star}$ \\
\hline Humed Relativa & $0.283^{\star \star \star}$ & ns & ns \\
\hline H. relativa -1 & $0.302^{\star \star \star}$ & $0.164^{\text {^ᄎ }}$ & ns \\
\hline H. relativa -2 & $0.310^{\text {kA }}$ & $0.197^{\star \star}$ & ns \\
\hline H. relativa -3 & $0.284^{\star \star \star}$ & $0.227^{\star \star \star}$ & ns \\
\hline Temp. máxima & $\mathbf{0 1 8 8 ^ { \star * }}$ & $0.481^{\star \star \star}$ & $0.182^{\text {* }}$ \\
\hline T. máxima -1 & ns & $0.421^{\star \star \star}$ & $0.214^{\star \star \star}$ \\
\hline T. máxima -2 & ns & $0.363^{\star \star \star}$ & $0.179^{\text {** }}$ \\
\hline T. máxima -3 & ns & $0.285^{\star \star \star}$ & $0.197^{\star \star}$ \\
\hline Temp. media & ns & ns & $0.152^{\star}$ \\
\hline T. media -1 & ns & ns & ns \\
\hline T. media -2 & ns & ns & ns \\
\hline T. media -3 & ns & ns & ns \\
\hline Temp. mínima & $0.250^{\star \star \star}$ & $0.255^{\star \star \star}$ & $0.247^{\star \star \star}$ \\
\hline T. mínima -1 & $0.245^{\star \star \star}$ & $0.160^{\star}$ & $0.206^{\star \star \star}$ \\
\hline T. mínima -2 & $0.244^{\star \star \star ~}$ & ns & $0.236^{\star \star \star}$ \\
\hline T. mínima-3 & $0182^{\star *}$ & ns & $0.158^{\star}$ \\
\hline Punto de rocío & $0.487^{\star \star \star}$ & ns & $0.375^{\star \star \star}$ \\
\hline P. de rocío - 1 & $0.456^{\star \star \star}$ & ns & $0.334^{\star \star \star}$ \\
\hline P. de rocío-2 & $0.430^{\star \star \star}$ & ns & $0.325^{\star \star \star}$ \\
\hline P. de rocío - 3 & 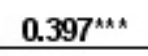 & ns & $0.322^{\star \star \star}$ \\
\hline
\end{tabular}




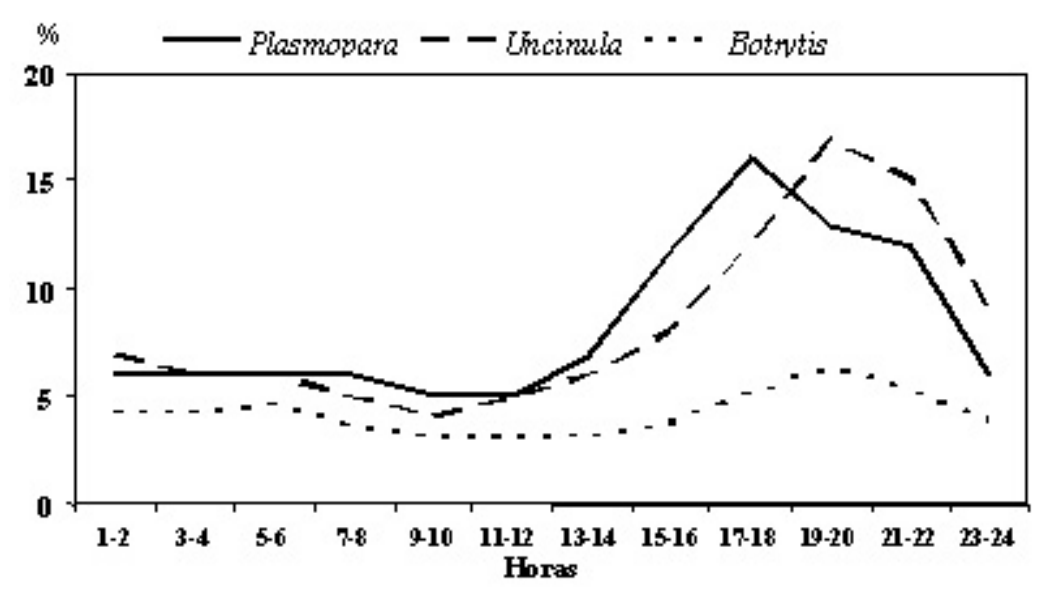

Figura 5.- Evolución intradiaria de la concentración de propágulos de Botrytis, Uncinula y Plasmopara

\section{DISCUSION}

Los resultados obtenidos en el estudio realizado en Cenlle, son similares a los señalados en viñedos de otras localidades de la comarca del Ribeiro en años anteriores (Díaz et al., 1997; 1998), así como los obtenidos en viñedos de la parte oriental de la provincia de Ourense (Díaz, 1999; Rodríguez-Rajo et al., 2002) pertenecientes a la Denominación de Origen de Valdeorras. De los tres propágulos fúngicos estudiados las concentraciones más elevadas pertenecen, en todos los casos a $\boldsymbol{B}$. cinerea, seguidas por las de $\boldsymbol{U}$. necator y finalmente por P. viticola, cuya presencia no se detectó en más de un tercio del total de días de este estudio. Según algunos autores las

Tabla 3.- Modelo de predicción de las concentraciones de conidios de Botrytis

\begin{tabular}{|c|c|c|c|c|c|c|c|}
\hline & $R$ & $R^{2}$ & Act. $r^{2}$ & $F$ & g. $l$ & $p$ & $\begin{array}{l}\text { En. Stand } \\
\text { Estimalos }\end{array}$ \\
\hline & 0.691 & 0.477 & 0.466 & 41.093 & 3.135 & 0.000 & 42.220 \\
\hline Botrytis & \multicolumn{7}{|c|}{ Botrytis $=-24.204+\left(0.707^{\star}\right.$ Botrytis -1$)+\left(2.995^{\star}\right.$ H. abs- 1$)+\left(0.524^{\star}\right.$ Punto rocio- 1$)$} \\
\hline \multirow[b]{3}{*}{ Intercpt } & & rr. Stau & & \multirow{2}{*}{\multicolumn{2}{|c|}{ Err. Stand. B }} & & \\
\hline & BETA & BETA & B & & & $\mathbf{t}(\mathbf{1 3 5})$ & p-level \\
\hline & & & -24.204 & \multicolumn{2}{|c|}{142.093} & -0.170 & 0.865 \\
\hline Botrytis- 1 & 0.658 & 0.066 & 0.707 & \multicolumn{2}{|c|}{0.071} & 9.971 & 0.000 \\
\hline H. absoluta-1 & 0.068 & 0.906 & 2.995 & \multicolumn{2}{|c|}{39.847} & 0.075 & 0.940 \\
\hline Punto rocio 1 & 0.020 & 0.903 & 0.524 & \multicolumn{2}{|c|}{23.431} & 0.022 & 0.982 \\
\hline
\end{tabular}

Tabla 4.- Modelo de predicción de las concentraciones de conidios de Uncinula

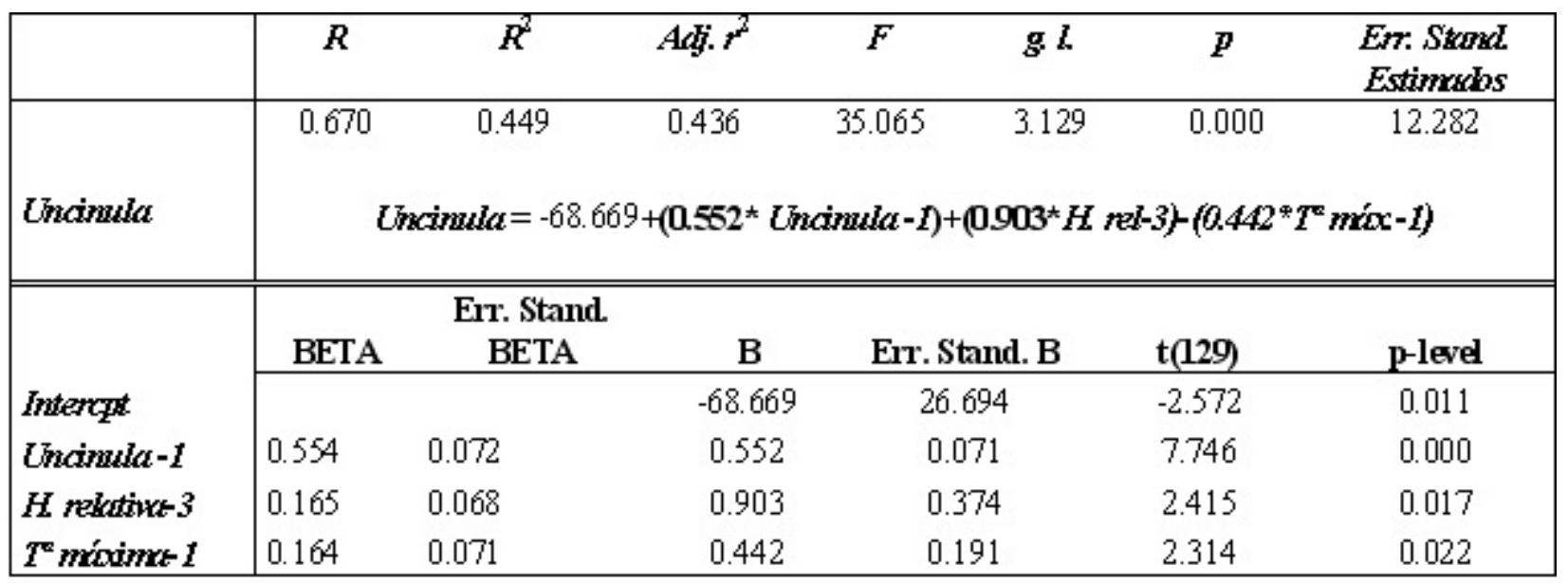


Tabla 5.- Modelo de predicción de las concentraciones de esporangios de Plasmopara

\begin{tabular}{|c|c|c|c|c|c|c|c|}
\hline & $R$ & $R^{2}$ & Adj. $\mathbf{r}^{2}$ & $F$ & g. $l$. & $\boldsymbol{p}$ & $\begin{array}{l}\text { Err. Stand. } \\
\text { Estimados }\end{array}$ \\
\hline & 0.392 & 0.154 & 0.135 & 8.191 & 3.135 & 0.000 & 2.8248 \\
\hline \multirow[t]{2}{*}{ Plasm opara } & \multicolumn{7}{|c|}{ Plasm opara $=-7.685+\left(0.293^{*}\right.$ Plasm. -1$)+\left(1590^{*}\right.$ H. abs -1$)-\left(0.6377^{*}\right.$ Punto rocío -1$)$} \\
\hline & BETA & $\begin{array}{r}\text { Err. Sta } \\
\text { BET A }\end{array}$ & B & Err. & nd. B & $t(135)$ & p-lev el \\
\hline Intercpt & & & -7.685 & & & -0.805 & 0.422 \\
\hline Plasm opara - 1 & 0.254 & 0.085 & 0.293 & & & 3.006 & 0.003 \\
\hline H. absoluta- 1 & 0.687 & 1.157 & 1.590 & & & 0.594 & 0.553 \\
\hline Punto rocio-1 & -0.467 & 1.153 & -0.637 & & & -0.405 & 0.686 \\
\hline
\end{tabular}

elevadas concentraciones de $\boldsymbol{B}$. cinerea podrían tener una acción antagónica de tipo nutricional o tóxica, llegando a ser capaces de eliminar de una forma eficaz la presencia de esporangios de P.viticola (Picco, 1992). Esta hipótesis podría ser aplicable en nuestro caso si observamos las gráficas de variación de las concentraciones de ambos propágulos a lo largo del período de estudio.

Las concentraciones más elevadas de conidios de Botrytis tuvieron lugar durante la segunda quincena de mayo y en concreto durante la fase de botones florales separados y comienzo de la floración. Pero al igual que ya se señaló en estudios anteriores (Rodríguez-Rajo et al., 2002), el pico máximo de concentración tiene lugar en los momentos previos a la vendimia, cuando las uvas se encuentran en plena maduración, atacando directamente a los racimos. Las concentraciones de conidios de Uncinula alcanzan sus máximos durante el mes de junio, cuando tienen lugar la floración y el cuajado, atacando a hojas y racimos. Finalmente las concentraciones de esporangios de Plasmopara en la atmósfera del viñedo, varían considerablemente a lo largo del período de estudio lo que podría ser consecuencia del tratamiento fitosanitario, que se aplicó 7 veces entre el 6 de mayo y el 8 de septiembre.

A lo largo del día los tres tipos de propágulos estudiados presentaron valores porcentuales mínimos durante la noche y las primeras horas de la mañana, para posteriormente incrementarse hasta niveles máximos durante la tarde. Al igual que en estudios realizados en otras zonas de Ourense (Rodríguez-Rajo et al., 2002), los picos de concentración se registraron entre las 19-20 h en el caso de Botrytis y Uncinula y en los casos de Plasmopara se encontraron a las 17-18 h, comportamiento muy diferente del observado en el mismo estudio (Rodríguez-Rajo et al., 2002) en el que se detectan por la mañana a las 11-12 h. Estas diferencias podrían ser debidas al bajo nivel de esporangios de Plasmopara en el viñedo de Cenlle.
Por otra parte la concentración de propágulos fúngicos en la atmósfera también se ve fuertemente influenciada por los parámetros meteorológicos, que afectan tanto a la capacidad de formación de nuevos cuerpos fructíferos como a la dispersión de los conidios y esporangios una vez formados. Un mismo factor meteorológico puede incidir de manera diferente, así por ejemplo, en el caso de Plasmopara, la presencia de gotas de lluvia resulta esencial para la germinación de las zoosporas de las que se originarán nuevos esporangios productores de zoosporas, pero no parecen tan importantes para su posterior dispersión, pudiendo incluso provocar la reducción de sus concentraciones por lavados de la atmósfera.

Si tenemos en cuenta el análisis de correlación entre las concentraciones de conidios de $\boldsymbol{B}$. cinerea y los diferentes parámetros meteorológicos durante el período de estudio, encontramos que la humedad, que influye directamente en todas las fases asexuales del desarrollo de este hongo y la temperatura de rocío, presentan la correlación positiva con coeficientes de regresión más elevados. La importancia de dichos factores para el desarrollo de este hongo ha sido señalada por diversos autores, lo que estaría de acuerdo con los resultados obtenidos. Así Broome et al. (1995) y English et al. (1989), apuntan que la germinación de estos conidios se produce como resultado de la interrelación entre la temperatura y la humedad y Marcilla (1968) y Ristori (1998), señalan que su germinación se activa con temperaturas entre $20^{\circ} \mathrm{C}$ y $25^{\circ} \mathrm{C}$.

Aunque con la precipitación no aparece ninguna correlación significativa en nuestro estudio, con la concentración de conidios de Botrytis, Gubler et al. (1985) y Bettiga et al. (1989), afirman que las precipitaciones del final de la estación incrementan la severidad de la podredumbre gris. En este sentido, en Cenlle, durante la primera quincena de septiembre se produce la mayor acumulación de agua recogida y a partir del día 10 de este 
mes se produce un acusado incremento en los niveles de conidios de Botrytis, registrándose los valores más elevados. La falta de correlación obtenida en nuestro caso probablemente se debe al bajo número de días en los que se produce la precipitación (36 días), por lo que la aplicación del test estadístico puede resultar no totalmente fiable.

La velocidad del viento del día anterior presenta correlación negativa con la concentración de conidios, ya que el aumento de la velocidad del viento favorece la dispersión atmosférica y por tanto provoca una disminución de la concentración de conidios en las proximidades de la fuente emisora (López et al., 1992), si bien favorece su transporte a mayor distancia por lo que induce la expansión de la enfermedad a otras zonas potenciales de desarrollar una infección secundaria.

La correlación entre los valores medios diarios de concentración de conidios de U.necator y las principales variables meteorológicas, presenta elevados coeficientes de correlación de signo positivo con la temperatura máxima y la humedad relativa, mientras que la correlación fue negativa con la precipitación. Según Smith et al. (1992), los factores meteorológicos que poseen una mayor influencia en el ciclo biológico del oidio son la humedad y la elevada temperatura, que es condición indispensable en el desarrollo de este ascomycete, y actuando el viento como un agente de dispersión. Correlaciones similares fueron encontradas en otros viñedos próximos a Cenlle, también en la cormarca del Ribeiro (Díaz et al., 1997; Díaz, 1999; Rodríguez-Rajo et al., 2002) .

En el caso de P.viticola, la correlación positiva con el coeficiente de correlación de Spearman más elevado, se obtuvo con la humedad absoluta y la temperatura de rocío. Por tanto, la humedad, parámetro íntimamente ligado a la precipitación, juega un papel importante en la variación de las concentraciones de esporangios de este oomycete. Según Hidalgo (1993), las condiciones meteorológicas que favorecen la contaminación primaria de este fitopatógeno son las precipitaciones superiores a $10 \mathrm{~mm}$ durante 1 ó 2 días consecutivos y acompañados de una temperatura media superior a los $12^{\circ} \mathrm{C}$. Para que se produzca una contaminación secundaria, solamente se requiere la presencia de lluvia para su diseminación. En nuestro estudio, pudimos comprobar como las mayores concentraciones tienen lugar en los días posteriores a los que se producen las precipitaciones más importantes. Valorando estos resultados, es posible que la precipitación tenga un doble efecto en la concentración de esporangios de este oomycete, la primera inmediata, produciendo un lavado atmosférico y arrastre de los esporangios que se encuentran en la superficie de los órganos infectados, y otra a medio plazo, favoreciendo su germinación.

Finalmente, y a pesar de las posibles alteraciones provocadas por la aplicación de tratamientos antifúngicos, se ha tratado de establecer para cada uno de los tipos fúngicos estudiados, modelos predictivos de la concentración de sus propágulos en la atmósfera del viñedo de Cenlle. Para ello se han tenido en cuenta las variables meteorológicas que alcanzaron el coeficiente de correlación más elevado y de signo positivo. Salvo en el caso de Plasmopara, el porcentaje de variación explicado es alto. Estudios llevados a cabo anteriormente (Rodríguez-Rajo et al., 2002) en otras zonas de la provincia de Ourense, señalan que las rectas de regresión propuestas para B.cinerea explican valores ligeramente inferiores (34\%) de la variabilidad de la concentración de conidios, aunque emplean como estimadores únicamente a la concentración de conidios del día anterior y la temperatura de rocío. Las rectas de regresión propuestas en el mismo trabajo para las concentraciones de Uncinula y Plasmopara no llegan a explicar un 10\% de la variabilidad de los propágulos fúngicos en el aire, mientras que en este caso estos porcentajes se elevan hasta un $43.6 \%$ y un $13.5 \%$ respectivamente.

Teniendo en cuenta lo anteriormente expuesto, en los viñedos del Ribeiro los propágulos más abundantes en la atmósfera durante el período vegetativo de la vid son los de B. cinerea, seguidas por $\boldsymbol{U}$. necator y finalmente P.viticola. Al igual que ocurre de forma estacional, la distribución de estos propágulos a lo largo del día no se produce de forma homogénea, siendo más elevados por la tarde. A la vista de la elevada variabilidad explicada por las rectas de regresión propuestas, los sistemas de control del nivel de propágulos combinados con parámetros meteorológicos como la temperatura de rocío o la humedad, suponen una gran mejoría en el control de estas enfermedades. La utilidad de estos modelos de predicción aumentaría con un mayor número de datos, por lo cual es necesario ampliar el estudio a años sucesivos.

\section{AGRADECIMIENTOS}

Los autores agradecen a la «Cooperativa Vitivinícola del Ribeiro» y en concreto a su Director Gerente D. Argimiro Levoso y a la Directora de Viticultura Uxía Quintela su colaboración y las facilidades proporcionadas para la ubicación del captador y la estación meteorológica. 


\section{REFERENCIAS}

Bettiga, L.J.; Gubler, W.D.; Arois, J.J. \& Bledsoe, A.M. (1989). Integrated control of Botrytis bunch rot of grape. Cal. Agricult. March-April: 9-11

Broome, J.C.; English, J.T.; Marois, J.J.; Latorre, B.A. \& Avilés, J.C. (1995). Development of an infection model for Botrytis bunch rot of grapes based on wetness duration and temperature. Phytopathology 85: 97-102

Díaz, M.R. (1999). Aplicación de la Aerobiología en la Agricultura. Control de enfermedades fúngicas \& producción de Vitis vinifera. Tesis doctoral. Universidad de Vigo.

Díaz, M.R.; Iglesias, I. \& Jato, V. (1997). Airborne concentrations of Botrytis, Uncinula and Plasmopara spores in Leiro-Ourense (NW Spain). Aerobiologia 13: 31-35

Díaz, M.R.; Iglesias, I. \& Jato, V. (1998). Seasonal variation of airborne fungal spores concentrations in a vinegard of Northwest Spain. Aerobiologia 14: 221-227

English, J.T.; Thomas, C.S.; Marois, J.J. \& Gubler, W.D. (1989). Microclimates of grapevine canopies associated with leaf removal and control of Botrytis bunch rot. Phytopathology 79: 395-401

Fernández, T. (2005). Control de la incidencia de Botrytis Cinerea en el cultivo de vid por métodos aerobiológicos. Proyecto fin de carrera Ingeniería Agroalimentaria. Universidad de Vigo.

Galán, C.; Tormo, R.; Cuevas, J.; Infante, F.; Domínguez, E. (1991). Theoretical dail\& variation patterns of airborne pollen in the South-west of Spain. Grana 30: 201-209
Gubler, W.D.; Marois, J.J.; Bledsoe, A.M. \& Bettiga, L.J. (1985). Control of Botrytis bunch rot of grape with canopy management. Plant Disease 71: 599-601

Hidalgo, L. (1993). Tratado de viticultura general. Editorial Mundi-Prensa, Madrid.

López, M.E.; Miñano, F.; Olive, J. \& Muñoz, A. (1992). La informática en la protección de los cultivos (Miniestaciones de alerta). Hojas divulgadoras del Ministerio de Agricultura, Pesca \& Alimentación. Secretaría General de Estructuras Agrarias, Madrid

Mansilla, J.P.; Pintos, C. \& Abelleira, A. (1991). Problemática fitosanitaria del viñedo en Galicia. Vitivinicultura 6:42-43

Marcilla, J. (1968). Tratado de Viticultura \& Enología Española. Tomo I. Ed. SAETA. Madrid

Picco, A.M. (1992). Presence in the atmosphere of vino and tomato patogens. Aerobiologia 8: 459-463

Ristori, P. (1998). La Botrytis o muffa grigia. Agronomia supp.4:2-4

Rodriguez-Rajo, F.J.; Seijo, M.C. \& Jato, V. (2002). Estudio de los niveles de los principales fitopatógenos par la optimización de cosechas de Vitis vinifera en Valedoras, Ourense (1998). Bot. Complot. 26:121-135

Smith, I.M.; Dunez, J.; Phillips, D.H.; Lelliot, R.A.; Archer, S.A. (1992). Manual de enfermedades de las plantas. Ediciones Mundi-Prensa, Madrid. 